\title{
Evaluation of acute and chronic pain outcomes after robotic, video-assisted thoracoscopic surgery, or open anatomic pulmonary resection
}

\author{
Sebastian T. Kwon, BS, ${ }^{a}$ Lili Zhao, PhD, ${ }^{b}$ Rishindra M. Reddy, MD, ${ }^{a}$ Andrew C. Chang, MD, \\ Mark B. Orringer, MD, ${ }^{\mathrm{a}}$ Chad M. Brummett, $\mathrm{MD},{ }^{\mathrm{c}}$ and Jules Lin, $\mathrm{MD}^{\mathrm{a}}$
}

\begin{abstract}
Objectives: Although robotic-assisted thoracic surgery (RATS) provides improved dexterity, the effect of RATS on pain compared with video-assisted thoracoscopic surgery (VATS) or open lobectomy is poorly understood. This study evaluated acute and chronic pain following RATS, VATS, and open anatomic pulmonary resection.
\end{abstract}

Methods: A retrospective review of 498 patients (502 procedures) who underwent RATS (74), VATS (227), and open (201) anatomic pulmonary resection including lobectomy and segmentectomy from 2010 to 2014 was performed to identify factors related to acute and chronic pain. Acute pain scores were analyzed over the first 9 postoperative days. Chronic pain was assessed using the validated PainDETECT survey.

Results: There were no significant differences in acute or chronic pain between RATS and VATS. There was a significant decrease in acute pain for patients with minimally invasive surgery $(P=.0004)$. Chronic numbness was significantly higher after open resection $(25.5 \%$ vs $11.6 \% ; P=.0269)$ but with no difference in other symptoms of chronic pain. Despite no significant difference in pain scores, $69.2 \%$ of patients who received RATS felt the approach affected pain versus $44.2 \%$ VATS $(P=.0330)$. On multivariable analysis, younger age $(P<.0001)$, female gender $(P=.0364)$, and baseline narcotic use $(P=.0142)$ were associated with acute pain, whereas younger age $(P=.0021)$ and major complications $(P=.0003)$ were associated with chronic numbness in patients who received MIS.

Conclusions: Although minimally invasive approaches resulted in less acute pain and chronic numbness, there were no significant differences between RATS and VATS. In contrast, more RATS patients believed the approach affected their pain, suggesting a difference between reality and perception. (J Thorac Cardiovasc Surg 2017;154:652-9)

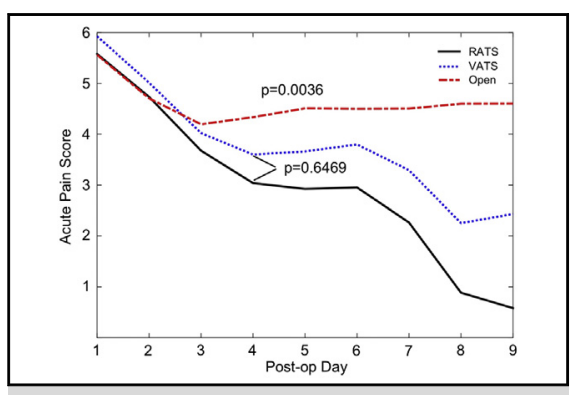

Pain scores were significantly higher after open but not between RATS or VATS lobectomy.

Central Message

Although robotics may provide improved dexterity, there were no differences in acute or chronic pain compared with thoracoscopic lobectomy.

\section{Perspective}

RATS lobectomy provides improved dexterity and visualization, but the effect of RATS on pain is poorly understood. Although minimally invasive lobectomy resulted in less acute pain and chronic numbness, there were no differences between RATS and thoracoscopic lobectomy. In contrast, more RATS patients believed the approach affected their pain, suggesting a difference between reality and perception.

See Editorial Commentary page 660.

See Editorial page 649

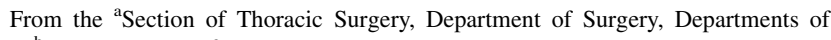
${ }^{\mathrm{b}}$ Biostatistics and ${ }^{\mathrm{c}}$ Anesthesiology, University of Michigan Medical School, Ann Arbor, Mich.

Funding was provided by the University of Michigan Summer Biomedical Research Program (to S.T.K.) and AATS Summer Intern Scholarship for Cardiothoracic Surgery (to S.T.K.).

Read at the 96th Annual Meeting of the American Association for Thoracic Surgery, Baltimore, Maryland, May 14-18, 2016.

Received for publication May 29, 2016; revisions received Jan 16, 2017; accepted for publication Feb 5, 2017; available ahead of print March 11, 2017.

Address for reprints: Jules Lin, MD, Section of Thoracic Surgery, University of Michigan Medical Center, 1500 E Medical Center Drive, 2120TC/5344, Ann Arbor, MI 48109-5344 (E-mail: juleslin@umich.edu).

$0022-5223 / \$ 36.00$

Copyright (c) 2017 by The American Association for Thoracic Surgery

http://dx.doi.org/10.1016/j.jtcvs.2017.02.008
Video-assisted thoracoscopic surgery (VATS) is a minimally invasive approach resulting in less tissue trauma, shorter recovery, and improved cosmesis. ${ }^{1}$ For early lung cancer, resection is the mainstay of treatment, and VATS lobectomy has become the treatment of choice..$^{2-4}$ There is a learning

Scanning this QR code will take you to a supplemental video and table for the article. 


\section{Abbreviations and Acronyms \\ ARDS $=$ acute respiratory distress syndrome \\ IASP $=$ International Association for the Study of Pain \\ IV $=$ intravenous \\ LOS = length of stay \\ MIS = minimally invasive surgery \\ PCA = patient-controlled analgesia \\ PD-Q = PainDETECT questionnaire \\ $\mathrm{POD}=$ postoperative day \\ RATS $=$ robotic-assisted thoracic surgery \\ STS $=$ Society of Thoracic Surgeons \\ VATS $=$ video-assisted thoracoscopic surgery}

curve to performing complex thoracoscopic procedures like lobectomy due to reduced tactile sensation. ${ }^{5}$ By combining 3-dimensional imaging and increased degrees of freedom, robotic surgery addresses some of these disadvantages. ${ }^{6,7}$ Although robotic-assisted thoracic surgery (RATS) may provide advantages in terms of dexterity, one of the disadvantages is a complete lack of tactile feedback, which could lead to tissue damage especially for inexperienced surgeons. ${ }^{8}$ Several studies have shown that RATS lobectomy can be performed safely by experienced thoracic surgeons with no significant difference in morbidity or mortality compared with VATS. ${ }^{9-11}$

However, the benefits of RATS compared with VATS lobectomy have not been clearly defined, especially with higher costs and longer operating times. ${ }^{10,12}$ One of the proposed advantages of robotic over thoracoscopic procedures is that the robotic arms rotate around a fulcrum point, which theoretically reduces torque on the chest wall decreasing damage to the intercostal nerves and surrounding tissues leading to reduced pain. Although RATS offers certain advantages for the surgeon, the benefits in terms of acute and chronic pain outcomes is less clear. ${ }^{13-15}$ This study evaluates postoperative acute and chronic pain outcomes after VATS, RATS, and open lobectomy.

\section{MATERIAL AND METHODS}

Approval from the University of Michigan Institutional Review Board was obtained. Consent was waived for the retrospective review of perioperative pain scores but was obtained when patients completed chronic pain surveys. Consecutive patients who underwent VATS, RATS, and open lobectomy or segmentectomy between February 2010 and June 2014 were included. Patients who underwent chest wall resection or previous ipsilateral thoracic surgery were excluded. Pre-resection mediastinoscopy and diagnostic wedge resection were not considered previous thoracic operations. Pulmonary resections were performed by 7 surgeons. Two surgeons performed all the RATS procedures but used VATS and RATS interchangeably depending on equipment availability with no preference based on size or location of the lesion. Although an open approach was favored for centrally located tumors, this represents the current practice in most thoracic groups.
VATS procedures were performed with a 3- or 4-port technique with an access incision less than $5 \mathrm{~cm}$ and no rib spreading. A 4-arm technique and an additional 12-mm port for the bedside assistant was used for RATS (Video 1). Posterolateral thoracotomies, generally serratus-sparing, were performed through the fifth interspace after shingling the sixth rib to facilitate rib spreading. All VATS and RATS port sites were infiltrated with $1 \%$ lidocaine $/ 0.25 \%$ bupivacaine with an intercostal nerve block and postoperative patient-controlled analgesia (PCA) transitioned to oral narcotics. Thoracotomy patients underwent epidural or paraspinous catheter placement supplemented with a PCA when needed.

\section{Data Collection}

A retrospective review of 498 patients who underwent 502 lobectomies or segmentectomies, including 74 RATS, 227 VATS, and 201 thoracotomies, was performed to identify factors related to postoperative pain (Table 1). Of these patients, 20 underwent thoracoscopic and 8 open segmentectomies. If a minimally invasive procedure was converted to thoracotomy, the patient was classified as having a thoracotomy. The Society of Thoracic Surgeons (STS) institutional database was queried along with the medical record.

To make the results comparable with other studies, standardized assessment tools were used. For acute ( $<10$ days) postoperative pain, visual pain scores, self-reported by the patient to the nurse, were collected. ${ }^{16}$ Focusing on the severity, the maximum pain scores from each postoperative day (POD) were taken from all patients and averaged using a generalized mixed model to create a progression of postoperative pain. Chronic pain is defined by the International Association for the Study of Pain (IASP) as having pain at the site of surgery more than 2 months afterward ${ }^{17}$ Symptoms of chronic pain were assessed using the validated PainDETECT questionnaire (PD-Q), which has been used previously to evaluate post-thoracotomy pain. ${ }^{18-20}$ If a patient failed to respond by mail after 2 attempts, up to 2 phone calls were made to administer a phone survey including questions from the PD-Q (Table E1). In the design stage, we specifically selected 11 variables (Tables 2 and 3) to be included in the survey because they were hypothesized to be potentially important factors for comparing surgical groups. In the analysis, we did not adjust for multiple comparisons because we set up a separate hypothesis for each of the 11 variables before we collected the data. Four patients underwent more than 1 lobectomy during different hospitalizations and had their inpatient courses analyzed separately for acute pain but were evaluated once for chronic pain at the time of the study.

Preoperative characteristics, including age, gender, tumor stage, preoperative narcotic use, and smoking status were collected. Major comorbidities included preoperative chemotherapy or radiation, coronary disease, peripheral vascular disease, cerebrovascular disease, pulmonary hypertension, diabetes, creatinine $>1.5$, dialysis, chronic obstructive pulmonary

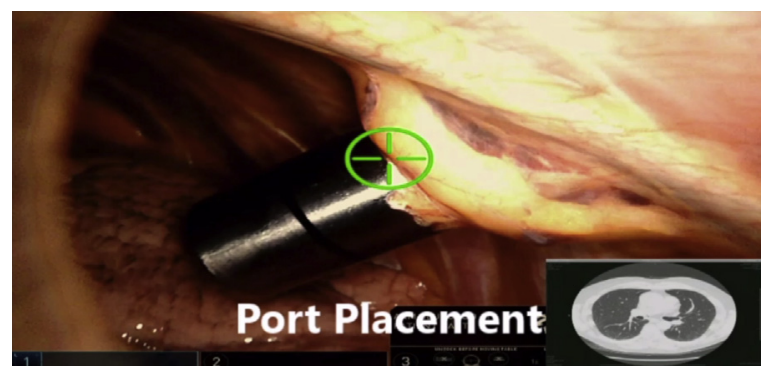

VIDEO 1. This brief video demonstrates the port placement and standard techniques used during a robotic-assisted thoracoscopic right upper lobectomy including the use of the robotic stapler and an intercostal nerve block. Video available at: http://www.jtcvsonline.org/article/S0022-5223(17) 30216-7/addons. 
TABLE 1. Patient characteristics

\begin{tabular}{|c|c|c|c|c|c|c|}
\hline Characteristic & All (502) & RATS (74) & VATS (227) & Open (201) & $\boldsymbol{P} *$ & $\boldsymbol{P}$ \\
\hline Mean age, y (range) & $65.7(18-93)$ & $66.7(37-91)$ & $65.4(18-87)$ & $65.7(20-93)$ & .9727 & .3940 \\
\hline Men & $247(49.2)$ & $28(37.8)$ & $106(46.7)$ & $113(56.2)$ & .0102 & .1834 \\
\hline Any major comorbidities & $285(57.2)$ & $42(56.8)$ & $109(48.0)$ & $134(66.7)$ & .0003 & .1922 \\
\hline Stage I/II & $368(73.3)$ & $59(79.7)$ & $178(78.4)$ & $131(65.2)$ & $<.0001$ & .3888 \\
\hline Stage III/IV & $86(17.1)$ & $6(8.1)$ & $11(4.8)$ & $42(20.9)$ & & \\
\hline Not lung primary & $48(9.6)$ & $9(12.2)$ & $38(16.7)$ & $28(13.9)$ & .6033 & .3455 \\
\hline Preoperative chemotherapy & $12(2.4)$ & $0(0)$ & $0(0)$ & $12(6.0)$ & $<.0001$ & \\
\hline Postoperative chemotherapy & $58(11.6)$ & $3(4.1)$ & $24(10.6)$ & $31(15.4)$ & .0267 & .0866 \\
\hline Preoperative radiation & $12(2.4)$ & $0(0)$ & $0(0)$ & $12(6.0)$ & $<.0001$ & \\
\hline Postoperative radiation & $15(3.0)$ & $0(0)$ & $4(1.8)$ & $11(5.5)$ & .0075 & .5753 \\
\hline Preoperative narcotic use & $90(17.9)$ & $11(14.9)$ & $30(13.2)$ & $49(24.4)$ & .0021 & .7184 \\
\hline Current smoker & $22(4.4)$ & $2(2.7)$ & $12(5.3)$ & $8(4.0)$ & .7184 & .5296 \\
\hline Quit smoking $>1$ mo & $366(72.9)$ & $54(73.0)$ & $156(68.7)$ & $156(77.6)$ & .0528 & .4884 \\
\hline Never smoker & $114(22.7)$ & $18(24.3)$ & $59(26.0)$ & $37(18.4)$ & .0603 & .7773 \\
\hline Prior thoracic operation & $3(0.6)$ & $0(0)$ & $0(0)$ & $3(1.5)$ & .0636 & \\
\hline Right side & $274(54.6)$ & $49(66.2)$ & $120(52.9)$ & $105(52.2)$ & .3897 & .0444 \\
\hline OR time, mean & 232 & 233 & 200 & 268 & $<.0001$ & .0016 \\
\hline Convert to open & 55 & $14(18.9)$ & $41(18.1)$ & - & & .8625 \\
\hline Prolonged air leak & $36(7.2)$ & $2(2.7)$ & $14(6.2)$ & $20(10.0)$ & .0535 & .3733 \\
\hline Chest tube (median days) & $3.0(1-49)$ & $3.0(2-49)$ & $3.0(1-39)$ & $4.0(1-41)$ & .0004 & .7508 \\
\hline In-hospital mortality & $1(0.2)$ & $0(0)$ & $0(0)$ & $1(0.5)$ & .4004 & \\
\hline Epidural & $117(23.3)$ & $1(1.4)$ & $13(5.7)$ & $103(51.2)$ & $<.0001$ & .2008 \\
\hline Paraspinous catheter & $64(12.7)$ & $0(0)$ & $0(0)$ & $64(15.9)$ & $<.0001$ & \\
\hline Median LOS, d (range) & $5.0(2-71)$ & $4.0(2-53)$ & $4.0(2-45)$ & $6.0(2-71)$ & $<.0001$ & .3807 \\
\hline Mean follow-up, d & 828 & 719 & 846 & 853 & .5890 & .0020 \\
\hline Deceased & $116(23.1)$ & $17(23.0)$ & $43(18.9)$ & $56(27.9)$ & .0390 & .4503 \\
\hline
\end{tabular}

Values are $\mathrm{n}(\%)$ unless otherwise indicated. Values in bold are significant at $P<.05$. RATS, Robotic-assisted thoracic surgery; VATS, video-assisted thoracoscopic surgery; OR, operating room; LOS, length of stay. *Minimally invasive surgery versus Open. $\dagger$ RATS versus VATS.

disease, pulmonary fibrosis, forced expiratory volume in 1 second $<50 \%$ predicted, diffusion lung capacity for carbon monoxide $<40 \%$ predicted, and a Zubrod score $\geq 2$. Epidural, paraspinous catheter, PCA, intravenous (IV) narcotics, and oral narcotic doses were recorded. Oral and IV narcotics were converted to morphine equivalents so the dosages could be compared.
Postoperative complications included return to the operating room, air leak, pneumonia, acute respiratory distress syndrome (ARDS), respiratory failure, bronchopleural fistula, pulmonary embolism, pneumothorax, ventilation, reintubation, tracheostomy, atrial or ventricular arrhythmia, myocardial infarction, deep venous thrombosis, empyema, sepsis, postoperative

TABLE 2. Chronic pain open versus MIS

\begin{tabular}{lccc}
\hline \multicolumn{1}{c}{ Variables } & MIS (112) & Open (55) & \multicolumn{1}{c}{$\boldsymbol{P}$} \\
\hline Pain & $26(23.2)$ & $18(32.7)$ & .1966 \\
Burning & $5(4.5)$ & $5(9.1)$ & .3001 \\
Tingling & $9(8.0)$ & $6(10.9)$ & .5714 \\
Prickling & $9(8.0)$ & $5(9.1)$ & .7755 \\
Light touch & $10(8.9)$ & $7(12.7)$ & .4301 \\
Numbness & $13(11.6)$ & $14(25.5)$ & $1(1.8)$ \\
Cold & $0(0)$ & $1(1.8)$ & .0269 \\
Heat & $0(0)$ & Yes $(50[90.9])$ & .3293 \\
Have surgery again? & Yes $(103[92.0])$ & No $(2[3.6])$ & .3293 \\
& No $(6[5.4])$ & Not sure $(3[5.5])$ & Yes $(20[36.4])$ \\
Approach affects pain? & Not sure $(3[2.7])$ & No $(18[32.7])$ \\
& Yes $(56[50.0])$ & Not sure $(17[30.9])$ \\
Affect pain how? & No $(45[40.2])$ & Positively $(11 / 19 ; 57.9 \%)$ \\
& Not sure $(11[9.8])$ & Negatively $(8 / 19 ; 42.1 \%)$ \\
\end{tabular}

Values are $\mathrm{n}(\%)$ unless otherwise indicated. Values in bold are significant at $P<.05 . M I S$, Minimally invasive surgery. 
TABLE 3. Chronic pain RATS versus VATS

\begin{tabular}{lccc}
\hline \multicolumn{1}{c}{ Variables } & RATS (26) & VATS (86) & \multicolumn{1}{c}{$\boldsymbol{P}$} \\
\hline Pain & $9(34.6)$ & $17(19.8)$ & .1831 \\
Burning & $2(7.7)$ & $3(3.5)$ & .3288 \\
Tingling & $3(11.5)$ & $6(7.0)$ & .4311 \\
Prickling & $3(11.5)$ & $6(7.0)$ & .4311 \\
Light touch & $5(19.2)$ & $5(5.8)$ & .0504 \\
Numbness & $3(11.5)$ & $10(11.6)$ & $0(0)$ \\
Cold & $0(0)$ & $0(0)$ & 1.0 \\
Heat & $0(0)$ & Yes $(80[93.0])$ \\
Have surgery again? & Yes $(23[88.5])$ & No $(3[3.5])$ \\
& No $(3[11.5])$ & Not sure $(3[3.5])$ & Yes $(38[44.2])$ \\
Approach affects pain? & Not sure $(0[0])$ & No $(37[43.0])$ \\
& Yes $(18[69.2])$ & Not sure $(11[12.8])$ \\
Affect pain how? & No $(8[30.8])$ & Positively $(36 / 38 ; 94.7 \%)$ \\
& Not sure $(0[0])$ & Negatively $(1 / 38 ; 2.6 \%)$
\end{tabular}

transfusion, ileus, delirium, and new renal failure. Minor complications were urinary tract infection, urinary retention, and surgical site infection. Respiratory complications included pneumonia, reintubation, respiratory failure, atelectasis, ARDS, mechanical ventilation, and tracheostomy.

\section{Statistical Analysis}

A generalized linear mixed model (PROC Glimmix) was used to estimate the longitudinal acute high pain scores using B-splines, and the pain scores were compared among the 3 surgical groups (RATS, VATS, and open). ${ }^{21,22}$ In the model, the group's main effect creates separate intercepts for each group, and the interaction of the group with the spline effect creates separate trends. This model allows us to compare the pain scores on each POD. The primary analysis for the pain scores was to compare the score trajectories between surgical groups, and there was no nonparametric test to perform this. Two-sample $t$ tests were used to compare acute pain scores, ${ }^{23}$ operative time, and length of hospital stay between RATS versus VATS and open versus minimally invasive surgery (MIS) (VATS or RATS). Chi-square or Fisher exact tests were used to evaluate categorical complications.

Multivariable outcomes of interest were acute pain and chronic numbness. A multivariable analysis was performed with the surgical approach as the primary variable. Covariates including age, smoking status, sex, neoadjuvant chemotherapy, neoadjuvant radiation, any major comorbidity, cancer stage, baseline narcotic use, average morphine used per day, paraspinous catheter, any major complication, epidural, prolonged air leak, acute pain, adjuvant chemotherapy, and adjuvant radiation were evaluated. A stepwise procedure was used to identify the most important factors with a significance level of .15 to select variables for the multivariable model. The surgical approach was always included. Significance was determined if $P$ values were $<.05$. Additionally, propensity score methods were used to compare surgical approaches after adjusting for the previously mentioned covariates. Specifically, we used inverse probability treatment weights and covariate adjustment using propensity scores. ${ }^{24}$ First, propensity scores were calculated from a logistic regression model with the surgical approach as the outcome and all the previously mentioned covariates as predictors, and then the propensity scores obtained in the first step were used in another logistic regression model with the acute pain or chronic numbness as the outcome and the surgical approach as the predictor. In the first method, each subject was weighted differently using the inverse of the propensity score (with appropriate normalization of the weights), and the propensity score was included as a covariate in the second method. All analyses were conducted using SAS (version 9.4; SAS Institute, Cary, NC).

\section{RESULTS \\ Patient Characteristics}

The mean operating room time was significantly longer with RATS compared with VATS (233 vs 200 minutes; $P=.0016)$ and open versus MIS (268 vs 208 minutes; $P<.0001)$ (Table 1$)$. The open group had significantly more men and more advanced cancers compared with MIS with a higher incidence of preoperative and postoperative chemotherapy and radiation. At least 1 major comorbidity was present in $66.7 \%(134 / 201)$ of open patients which was significantly higher $(P=.0003)$ than RATS $(56.8 \%)$ or VATS $(48 \%)$. Median chest tube duration was longer, more patients used preoperative narcotics, and there was increased use of an epidural or paraspinous catheter.

There was no difference in length of stay (LOS) between RATS and VATS $(P=.3807)$ nor was there a difference in intensive care unit admission rates $(P=.7789)$. Open patients had a longer LOS (4.83 days MIS vs 7.31 days open; $P<.0001)$ and were more likely to be admitted to the intensive care unit $(6.0 \%$ [18/301] MIS vs $22.9 \%$ [46/ $201]$ open; $P<.0001)$. Rates of pneumonia $(3.0 \%$ [9/301] MIS vs $7.0 \%(14 / 201)$ open; $P=.0487)$ and reintubation (2.0\% [6/301] MIS vs $6.0 \%$ [12/201] open; $P=.0259)$ were also significantly higher after thoracotomy.

\section{Acute Pain}

There were no significant differences in acute pain between VATS and RATS (Figure 1). Although the RATS pain course appeared to be slightly decreased, especially in the later PODs, the difference was not significant 


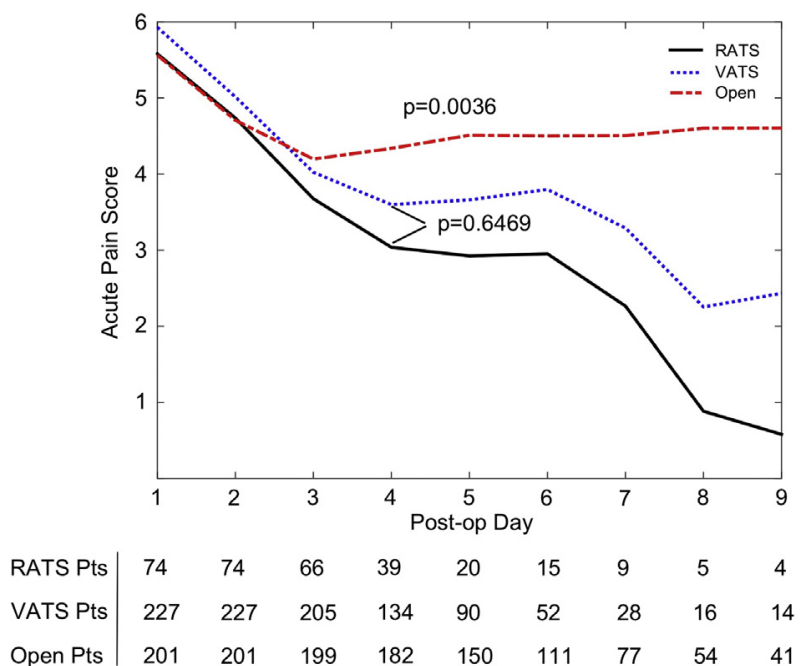

FIGURE 1. Daily high pain scores were significantly higher after open lobectomy compared with a minimally invasive approach (video-assisted thoracoscopic surgery $[V A T S]+$ robotic-assisted thoracic surgery $[R A T S]$ ), but only after postoperative day (POD) 4 . Pain scores were not significantly different between RATS and VATS. The number of patients in the hospital on each POD is included below as well as the lower and upper bound of the $95 \%$ confidence interval for the difference between groups. Values in bold are significant at $P<.05$. MIS, Minimally invasive surgery.

\begin{tabular}{|c|c|c|c|c|}
\hline & \multicolumn{2}{|c|}{ Acute pain score difference between RATS vs VATS } & \multicolumn{2}{|c|}{ Acute pain score difference between MIS vs open } \\
\hline & Confidence interval & $\boldsymbol{P}$ & Confidence interval & $\boldsymbol{P}$ \\
\hline POD1 & -1.0491 to 0.3522 & .3294 & -0.7768 to 0.2096 & .2596 \\
\hline POD2 & -0.8575 to 0.3002 & .3452 & -0.6298 to 0.1624 & .2474 \\
\hline POD3 & -0.9258 to 0.2341 & .2423 & -0.1411 to 0.6508 & .2070 \\
\hline POD4 & -1.3696 to 0.2447 & 1719 & 0.3810 to 1.3176 & .0004 \\
\hline POD5 & -1.6257 to 0.1500 & .1033 & 0.5030 to 1.4892 & $<.0001$ \\
\hline POD6 & -2.1261 to 0.4178 & .1950 & 0.1855 to 1.5872 & .0132 \\
\hline POD7 & -2.4716 to 0.4178 & .1634 & 0.6688 to 2.2302 & .0003 \\
\hline POD8 & -4.0033 to 1.2640 & .3078 & 1.3177 to 3.9896 & .0001 \\
\hline POD9 & -5.3282 to 1.6299 & .2959 & 0.8405 to 4.2324 & .0034 \\
\hline
\end{tabular}

$(P=.6469)$. On the other hand, there was a significant decrease in acute pain after MIS compared with thoracotomy starting on POD 4, (Figure 1; $P=.0004$ ). After excluding patients with an epidural, acute pain scores between MIS and open became significantly different even earlier on POD $2(P=.0003)$. On univariate analysis, major complications $(P=.0110)$ and baseline narcotic use $(P<.0001)$ correlated with acute pain, whereas modifier 22 , neoadjuvant chemotherapy and radiation, and previous thoracic operations did not. Patients with higher acute pain scores had higher postoperative oxygen requirements $(P<.0001)$.

\section{Chronic Pain}

Chronic pain surveys were completed by $86.7 \%$ (169/ 195) of patients who were alive and could be contacted at the time of the study (26 RATS, 87 VATS, and 56 open). Most patients (161/169 [95.3\%]) completed the validated PainDETECT survey, and 167 patients answered the questions on whether the surgical approach affected their pain. Eight patients completed the phone survey alone (which also contained the questions from the PainDETECT; Table E1). The differences in chronic pain between MIS and thoracotomy were not significantly different (23.2\% [26/112] MIS vs 32.7\% [18/55] open; $P=.1966)$. However, when evaluating individual symptoms, chronic numbness was significantly more common after open lobectomy $(11.6 \%$ [13/112] MIS vs $25.5 \%$ $[14 / 55]$ open; $P=.02)$. There was also a significant difference in the degree of numbness scored from 0 to 5 , with a mean of 1.61 for the MIS patients and 2.23 after thoracotomy $(P=.0264)$. Open patients were more likely to have other symptoms of chronic neuropathic pain, including burning, tingling, and pain with light touch, cold, or heat; however, these differences were not significant (Table 2).

Open patients were more likely to experience persistent pain with pain attacks (Pain course 2 on the PD-Q) $(2.6 \%$ [1/38] MIS vs $27.8 \%$ [5/18] open; $P=.0106$ ), whereas MIS patients experienced pain attacks without pain 
TABLE 4. Postoperative outcomes-multivariable analysis $(P$ values $)$

\begin{tabular}{lcc}
\hline \multicolumn{1}{c}{ Variables } & Acute pain & Chronic numbness \\
\hline RATS vs VATS variables & & \\
Procedure type & .6787 & .2593 \\
Sex & $\mathbf{. 0 3 6 4}$ & $>.15$ \\
Age & $<.0001$ & $\mathbf{. 0 0 2 1}$ \\
Baseline narcotic & $\mathbf{. 0 1 4 2}$ & $>.15$ \\
Major comorbidity & $>.15$ & .1382 \\
Major complication & .0652 & $\mathbf{. 0 0 0 3}$ \\
Average morphine/d & $\mathbf{. 0 0 2 7}$ & $>.15$ \\
MIS vs Open variables & & \\
Procedure type & .6316 & $\mathbf{. 0 1 1 8}$ \\
Sex & $<.0001$ & $>.15$ \\
Age & $<.0001$ & $\mathbf{. 0 0 0 2}$ \\
Baseline narcotic & $\mathbf{. 0 0 0 8}$ & $>.15$ \\
Major comorbidity & $>.15$ & .0847 \\
Major complication & .1515 & $\mathbf{. 0 1 0 5}$ \\
Average morphine/d & $\mathbf{. 0 0 0 1}$ & $>.15$ \\
\hline Only variables with $P<.15$ are shown. Values in bold are significant at $P<.05$. See \\
Methods section for a full list of variables. $R A T S$, Robotic-assisted thoracic surgery; \\
VATS, video-assisted thoracoscopic surgery; MIS, minimally invasive surgery.
\end{tabular}

between, although this was not significant (Pain course 3 ; $65.8 \%$ [25/38] MIS vs 38.9\% [7/18] open; $P=.0837$ ). Younger patients were significantly more likely to have chronic numbness $(P=.0004)$. For open patients, $36.4 \%$ (20/55) felt the approach affected their pain versus $50.0 \%$ $(56 / 112)$ MIS $(P=.0040)$ and were more likely to feel that the approach affected their pain negatively $(42.1 \%$ [8/19] open vs $3.6 \%$ [2/55] MIS; $P=.0002$ ).

Although RATS patients had a higher rate of chronic neuropathic pain, this was not significant $(34.6 \%$ [9/26] RATS vs $19.8 \%$ [17/86] VATS; $P=.1831$ ) (Table 3 ). However, significantly more RATS patients felt that the approach affected their pain $(69.2 \%$ [18/26] RATS vs $44.2 \%$ [38/86] VATS; $P=.0330)$, and most $(88.2 \% ; 15 /$ 17) thought that the approach affected their pain positively. Of the VATS patients who felt the approach affected their pain, 94.7\% (36/38) also felt that the approach affected their pain positively.

\section{Pain Control Modalities}

Patients requiring oral or IV narcotics had higher pain scores (5.0961 vs $2.3865 ; P<.0001)$. Morphine equivalents also positively correlated with pain scores $(P<.0001)$. There was no difference in the average morphine equivalents between RATS and VATS patients $(P=.7552)$ or MIS and open patients $(P=.6892)$. However, when morphine equivalents were separated by postoperative day, after excluding patients with epidurals, open patients used significantly more morphine equivalents from POD3 through POD6 $(P<.0001,<.0001, .0007$, and .0060, respectively). Patients with a paraspinous catheter had significantly higher pain scores $(4.5000$ vs 5.1307; $P=$.0009).

\section{Multivariable Analysis}

For multivariable analysis, outcomes evaluated included median acute pain scores and chronic numbness (Table 4). For RATS versus VATS, younger age $(P<.0001)$, baseline narcotic use $(P=.0142)$, increased average morphine per day $(P=.0027)$, and female gender $(P=.0364)$ were associated with increased acute pain scores, whereas the approach and major complications were not. Chronic numbness was associated with the occurrence of any major complication $(P=.0003)$ and younger age $(P=.0021)$ but not procedure type or postoperative acute pain score.

Comparing open versus MIS, younger age $(P<.0001)$, female gender $(P<.0001)$, baseline narcotic use $(P=.0008)$, and increased average morphine per day $(P=.0001)$ were associated with increased acute pain. Chronic numbness was associated with younger age (.0002), thoracotomy (odds ratio $3.543 ; 95 \%$ confidence interval $[\mathrm{CI}] 1.323-0.486 ; P=.0118$ ), and any major complication $(P=.0105)$. Smoking, neoadjuvant chemotherapy or radiation, stage, paraspinous catheter, epidural, prolonged air leak, acute pain, and adjuvant chemotherapy or radiation were not significant for RATS versus VATS or open versus MIS.

\section{Propensity Scoring}

Using 2 propensity scoring methods, RATS versus VATS $(P=.49$ and .31$)$ and open versus MIS (0.72 vs 0.68$)$ were not significantly different for acute pain. For chronic numbness, RATS versus VATS was not significantly different $(P=1$ and .65), whereas MIS versus open was $(P<.0001$ and 0.0119$)$. The odds ratios were $6.82(95 \%$ CI 2.89-16.1) and 5.16 (95\% CI 1.44-18.52) using the 2 propensity scoring methods.

\section{DISCUSSION}

This is the first study evaluating acute and chronic pain outcomes after RATS, VATS, and open anatomic lung resections using validated pain assessment tools. Nasir et $\mathrm{al}^{25}$ reported a median pain score of 2/10 in 394 patients undergoing robotic lung resection 3 weeks postoperatively, but no perioperative pain data were provided, and the only comparison group was 41 patients converted to thoracotomy. Although Chiu et $\mathrm{al}^{26}$ found lower pain scores after robotic versus laparoscopic hysterectomy, Park et $\mathrm{al}^{27}$ found no significant difference in a randomized trial of 71 patients undergoing robotic versus laparoscopic right colectomy.

Our results showed no significant difference between VATS and RATS for acute or chronic pain outcomes. However, more RATS patients $(69.2 \%)$ felt that the robotic approach affected their pain, and $88.2 \%$ felt the affect was positive, suggesting a difference between reality and perception likely reflecting successful marketing. When discussing open versus MIS approaches, surgeons often 
describe less pain and quicker recovery after MIS, which could affect patients' perspectives. However, no difference in pain was described by our surgeons when counseling patients on VATS versus RATS.

While acute pain data were collected over the initial 9 PODs, many MIS patients were discharged before day 9 . With a median LOS of 4 days, the MIS patients still in the hospital likely had complications, such as a prolonged air leak or pneumonia, and MIS patients at home may have had lower pain scores, potentially increasing the difference in acute pain seen between open and MIS.

Female gender was an independent predictor of increased acute pain in VATS versus RATS and open versus MIS patients, suggesting that female patients should be more aggressively treated for acute pain, using narcotic patches or an epidural. Acute pain scores were also higher from POD 3 to 6 after thoracotomy, reflecting discontinuation of most epidurals on POD 3. When indicated, it may be beneficial to keep epidurals until POD 5 to improve pain control and to use adjuvant methods such as narcotic patches once the epidural is removed.

Although previous studies have associated higher postoperative pain with smoking, there was no significant relationship in the current study. However, our institution has a lower percentage of current smokers because patients are strongly encouraged to quit smoking preoperatively to decrease pulmonary complications with only $4.4 \%$ of patients smoking at the time of surgery and no significant difference between groups.

Nomori et $\mathrm{al}^{28}$ found no significant difference in chronic pain among VATS, limited thoracotomy, or open thoracotomy. Open thoracotomy was associated with significantly higher acute pain. Maguire et $\mathrm{al}^{29}$ evaluated 600 patients who underwent VATS or thoracotomy and found chronic pain in $57 \%$ at 7 to 12 months. Younger age was associated with chronic pain, whereas the approach was not. In a study of 144 VATS patients, Hutter et $\mathrm{al}^{30}$ reported chronic pain in $20.1 \%$ with $16.9 \%$ experiencing numbness and $8.3 \%$ dysesthesia. Grosen et $\mathrm{al}^{31}$ evaluated 414 patients undergoing thoracotomy for lung cancer and found chronic pain in $19 \%$.

Our results showed chronic pain in $34.6 \%$ after RATS, $19.8 \%$ after VATS, and $32.7 \%$ after thoracotomy; however, the differences were significant only for chronic numbness in open versus MIS $(P=.0269)$. Although neuropathic pain is not uncommon after thoracic surgery, Steegers et $\mathrm{al}^{19}$ found that only half of chronic pain included a neuropathic component. Numbness is a standard measure of chronic pain on the PainDETECT questionnaire, which has been used previously to evaluate post-thoracotomy pain. ${ }^{18,19}$ Although use of both mailed and phone surveys could introduce variation, the phone survey included the same questions from the PainDETECT, and the vast majority (161/169 [95.3\%]) completed the PainDETECT with only 8 patients completing the phone survey alone.
Although thoracotomy was favored for central tumors, which could bias the results, we believe this represents current practice in most thoracic groups. VATS lobectomy has become the standard approach for most lung cancers, and a randomized trial would be impossible. Although thoracotomy was associated with longer operative times, RATS also took significantly longer than VATS with no significant difference in pain outcomes. Although there were differences in cancer stage between open and MIS, we have attempted to control for this by including these variables in our multivariable analysis, and thoracotomy was independently predictive of chronic numbness.

For postoperative complications after MIS, the only significant difference was a higher rate of urinary retention in RATS versus VATS. This was possibly due to significantly longer operating times because the length of anesthesia has been correlated with voiding times. ${ }^{32}$ Our approach to Foley removal within 24 hours is the same for RATS and VATS. For open patients, urinary retention may not have correlated with procedure length because the Foley remained in place until removal of the epidural. In contrast, pulmonary complications, including pneumonia and reintubation, were significantly more common after thoracotomy, likely related to increased postoperative pain.

With any retrospective study, there are limitations. Chronic pain data were collected at the time of the study instead of specific time points, which could result in recall bias. However, previous studies have used a similar approach including a VATS study by Hutter et al ${ }^{17,30}$ evaluating patients at least 2 months postoperatively, which is the definition of chronic pain used by the IASP.

Although epidurals were routinely used for thoracotomy, they were infrequent after MIS. A prospective study using a standardized pain regimen in all patients would be needed to address this limitation. However, one of the aims was to evaluate pain outcomes in real-life conditions. Although epidurals are standard after thoracotomy, ${ }^{33}$ only $10 \%$ to $46 \%$ received an epidural after VATS. ${ }^{34}$ In addition, there was no significant difference in pain scores in a randomized trial between epidural and PCA after VATS lobectomy. ${ }^{35}$

Although epidurals are a potential confounding factor, epidurals would be expected to improve pain in open patients and decrease any differences between the 2 groups. Despite epidurals, there was still a significant difference in acute pain starting on POD 4, consistent with the removal of most epidurals on POD 3. Eliminating epidurals would likely increase the differences in acute pain, and excluding patients with an epidural revealed a significant difference in acute pain scores even earlier on POD 2. In addition, one of the main aims was to compare RATS and VATS, for which the pain control was the same with no epidurals.

Although there was variability in the frequency that scores were recorded, only the maximum score for each day was used to focus on the severity of postoperative 
pain. Although there are limitations in a retrospective study in confirming the consistency of pain assessments, the visual pain scale is used widely across specialties and institutions, and the pain assessment was similar between groups because all patients were cared for on the same unit by the same group of thoracic nurses during the same period.

The benefits of RATS lobectomy in terms of acute and chronic pain outcomes is unclear. Results of the current study show that although minimally invasive approaches resulted in less acute pain and chronic numbness, there were no significant differences between RATS and VATS. In contrast, more RATS patients believed the approach affected their pain, suggesting a difference between reality and perception. This study should serve as a basis for future larger, prospective studies evaluating acute and chronic pain outcomes after thoracic surgery.

\section{Conflict of Interest Statement}

Dr Reddy and Dr Lin: Intuitive Surgical Site Mentor and Speaker. Dr Brummett: Tonix Pharmaceuticals and Neuros Medical Inc. All other authors have nothing to disclose with regard to commercial support.

We thank Shari Barnett for her help with data collection for this project.

\section{References}

1. Nagahiro I, Andou A, Aoe M, Sano Y, Date H, Shimizu N. Pulmonary function, postoperative pain, and serum cytokine level after lobectomy: a comparison of VATS and conventional procedure. Ann Thorac Surg. 2001;72:362-5.

2. Grogan EL, Jones DR. VATS lobectomy is better than open thoracotomy: what is the evidence for short-term outcomes? Thorac Surg Clin. 2008;18:249-58.

3. Whitson BA, Groth SS, Duval SJ, Swanson SJ, Maddaus MA. Surgery for earlystage non-small cell lung cancer: a systematic review of the video-assisted thoracoscopic surgery versus thoracotomy approaches to lobectomy. Ann Thorac Surg. 2008;86:2008-16; discussion 2016-8.

4. Yan TD, Black D, Bannon PG, McCaughan BC. Systematic review and metaanalysis of randomized and nonrandomized trials on safety and efficacy of video-assisted thoracic surgery lobectomy for early-stage non-small-cell lung cancer. J Clin Oncol. 2009;27:2553-62.

5. Lanfranco AR, Castellanos AE, Desai JP, Meyers WC. Robotic surgery: a current perspective. Ann Surg. 2004;239:14-21.

6. Moorthy K, Munz Y, Dosis A, Hernandez J, Martin S, Bello F, et al. Dexterity enhancement with robotic surgery. Surg Endosc. 2004;18:790-5.

7. Morgan JA, Ginsburg ME, Sonett JR, Morales DL, Kohmoto T, Gorenstein LA, et al. Advanced thoracoscopic procedures are facilitated by computer-aided robotic technology. Eur J Cardiothorac Surg. 2003;23:883-7; discussion 887.

8. Bodner JC, Zitt M, Ott H, Wetscher GJ, Wykypiel H, Lucciarini P, et al. Roboticassisted thoracoscopic surgery (RATS) for benign and malignant esophageal tumors. Ann Thorac Surg. 2005;80:1202-6.

9. Cao C, Manganas C, Ang SC, Yan TD. A systematic review and meta-analysis on pulmonary resections by robotic video-assisted thoracic surgery. Ann Cardiothorac Surg. 2012;1:3-10.

10. Swanson SJ, Miller DL, McKenna RJ Jr, Howington J, Marshall MB, Yoo AC, et al. Comparing robot-assisted thoracic surgical lobectomy with conventional video-assisted thoracic surgical lobectomy and wedge resection: results from a multihospital database (Premier). J Thorac Cardiovasc Surg. 2014;147:929-37.

11. Park BJ, Melfi F, Mussi A, Maisonneuve P, Spaggiari L, Da Silva RK, et al. Robotic lobectomy for non-small cell lung cancer (NSCLC): long-term oncologic results. J Thorac Cardiovasc Surg. 2012;143:383-9.

12. Augustin F, Bodner J, Maier H, Schwinghammer C, Pichler B, Lucciarini P, et al, Robotic-assisted minimally invasive vs. thoracoscopic lung lobectomy: comparison of perioperative results in a learning curve setting. Langenbecks Arch Surg. 2013;398:895-901.

13. Flores RM, Alam N. Video-assisted thoracic surgery lobectomy (VATS), open thoracotomy, and the robot for lung cancer. Ann Thorac Surg. 2008;85:S710-5.

14. Park BJ, Flores RM, Rusch VW. Robotic assistance for video-assisted thoracic surgical lobectomy: technique and initial results. J Thorac Cardiovasc Surg. 2006;131:54-9.

15. Veronesi G, Galetta D, Maisonneuve P, Melfi F, Schmid RA, Borri A, et al. Fourarm robotic lobectomy for the treatment of early-stage lung cancer. J Thorac Car diovasc Surg. 2010;140:19-25.

16. Scott J, Huskisson EC. Graphic representation of pain. Pain. 1976;2:175-84.

17. Classification of chronic pain. Descriptions of chronic pain syndromes and definitions of pain terms. Prepared by the International Association for the Study of Pain, Subcommittee on Taxonomy. Pain Suppl. 1986;3:S1-226.

18. Kampe S, Lohmer J, Weinreich G, Hahn M, Stamatis G, Welter S. Epidura analgesia is not superior to systemic postoperative analgesia with regard to preventing chronic or neuropathic pain after thoracotomy. J Cardiothorac Surg. 2013;8:127.

19. Steegers MA, Snik DM, Verhagen AF, van der Drift MA, Wilder-Smith OH. Only half of the chronic pain after thoracic surgery shows a neuropathic component. $J$ Pain. 2008;9:955-61.

20. Freynhagen R, Baron R, Gockel U, Tolle TR. painDETECT: a new screening questionnaire to identify neuropathic components in patients with back pain Curr Med Res Opin. 2006;22:1911-20.

21. Bde Boor C. A Practical Guide to Splines, Revised Edition. New York, NY Springer-Verlag; 2001

22. Eilers PHC, Marx BD. Flexible smoothing with B-splines and penalties. Statistical Science. 1996;11:89-121.

23. Mantha S, Thisted R, Foss J, Ellis JE, Roizen MF. A proposal to use confidence intervals for visual analog scale data for pain measurement to determine clinical significance. Anesth Analg. 1993;77:1041-7.

24. Austin PC. An introduction to propensity score methods for reducing the effects of confounding in observational studies. Multivariate Behav Res. 2011;46: 399-424.

25. Nasir BS, Bryant AS, Minnich DJ, Wei B, Cerfolio RJ. Performing robotic lobectomy and segmentectomy: cost, profitability, and outcomes. Ann Thorac Surg. 2014;98:203-8; discussion 208-9.

26. Chiu LH, Chen CH, Tu PC, Chang CW, Yen YK, Liu WM. Comparison of robotic surgery and laparoscopy to perform total hysterectomy with pelvic adhesions or large uterus. J Minim Access Surg. 2015;11:87-93.

27. Park JS, Choi GS, Park SY, Kim HJ, Ryuk JP. Randomized clinical trial of robotassisted versus standard laparoscopic right colectomy. Br J Surg. 2012;99: 1219-26.

28. Nomori H, Cong Y, Sugimura H. Limited thoracotomy for segmentectomy: a comparison of postoperative pain with thoracoscopic lobectomy. Surg Today. 2016;46:1243-8

29. Maguire MF, Ravenscroft A, Beggs D, Duffy JP. A questionnaire study investigating the prevalence of the neuropathic component of chronic pain after thoracic surgery. Eur J Cardiothorac Surg. 2006;29:800-5.

30. Hutter J, Miller K, Moritz E. Chronic sequels after thoracoscopic procedures for benign diseases. Eur J Cardiothorac Surg. 2000;17:687-90.

31. Grosen K, Laue Petersen G, Pfeiffer-Jensen M, Hoejsgaard A, Pilegaard HK Persistent post-surgical pain following anterior thoracotomy for lung cancer: a cross-sectional study of prevalence, characteristics and interference with functioning. Eur J Cardiothorac Surg. 2013;43:95-103.

32. Mulroy MF, Salinas FV, Larkin KL, Polissar NL. Ambulatory surgery patient may be discharged before voiding after short-acting spinal and epidural anesthesia. Anesthesiology. 2002;97:315-9.

33. Joshi GP, Bonnet F, Shah R, Wilkinson RC, Camu F, Fischer B, et al. A systematic review of randomized trials evaluating regional techniques for postthoracotomy analgesia. Anesth Analg. 2008;107:1026-40.

34. Kaplowitz J, Papadakos PJ. Acute pain management for video-assisted thoracoscopic surgery: an update. J Cardiothorac Vasc Anesth. 2012;26:312-21.

35. Kim JA, Kim TH, Yang M, Gwak MS, Kim GS, Kim MJ, et al. Is intravenous patient controlled analgesia enough for pain control in patients who underwent thoracoscopy? J Korean Med Sci. 2009;24:930-5.

Key Words: acute pain, chronic pain, lobectomy, thoracoscopy, robotic surgery, thoracotomy 
TABLE E1. Phone survey

Postoperative pain and patient satisfaction

1. Over the past week have you had pain related to your surgery? (Y/N)

a. If yes, where is the pain located? (surgical site or specific location):

b. How would you characterize the pain? (circle all that apply):
i. Burning
ii. Tingling
iii. Prickling
iv. Light touching painful
v. Numbness
vi. Cold is painful
vii. Heat is painful

2. From a pain respect alone, would you do the surgery again? (Y/N)

3. Do you feel like the surgical approach (robotic, thoracoscopic, or open surgery) affected your postoperative pain $(\mathrm{Y} / \mathrm{N})$ ?

a. If yes, positively or negatively? 